\title{
CELL DIVISION
}

\section{Running rings around the spindle}

\begin{abstract}
When cells divide, each chromosome is duplicated and ane copy is passed to eachdaughter cell. The details of how these duplicated chromosomes are separated are still being puzzled out, but elsewhere in this issue, Georjana Bames and colleagues (Nature 440, 565-569; 2006) add a large piece to

the jigsaw.

During celldivision, an array of tiny filamentscalledmicrotubules spreads from the poles of the cell to the central plane, where the duplicated chromosomes congregate. This microtubule structure - the 'mitotic spindle' is responsible for dragging the two copies of the chromosome apart int othe two regions that will form the daughter cells. Microtubules are linear polymers of the protein tubulin of diameter $24 \mathrm{~nm}$ that grow or shrink astubulinmonomers are added to or lost from their ends. During chromosome segregation,
\end{abstract}

the microtubules depolymerize, and thus shorten, from the end nearest the central plane of the cell. So, as the spindle shrinks, the attached chromosomes are carried towards the pole.

The chromosames stick to the depolymerizing ends of the spindle microtubules through specialized protein complexes known as kinetochares. But if these ends are continually dissolving as the microtubules pull back, how do the chromosomes stay attached? The answer lies with a subcomplex in the kinetochore, called the Dam1 complex, which assembles into rings and paired helices that encircle the ends of depolymerizing microtubules (pictured).

Bameset al. used real-time imaging to watch the Daml complex on dynamic microtubules in vitro. They labelled the Dam1 complex and themicrotubules with different coloured fluorophores. As the

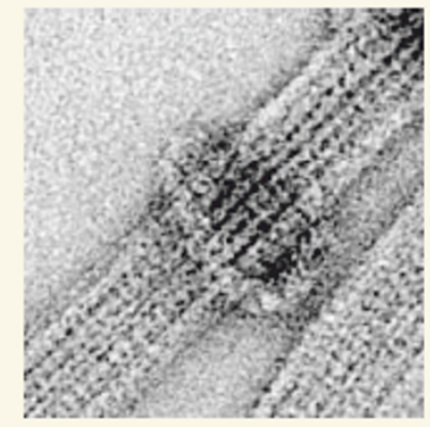

microtubules shortened during depolymerization, the Dam1 complex could clearly be seen progressing along the microtubules. Thismovement could be explained either by a 'sliding' mechanism, in which the Daml complex is continually associated with the microtubule lattice, or by a 'turnover' mechanism, withnew subunits of the Daml complex rapidly incorporating into existing rings as old subunits leave, thus propelling the ring along the microtubule.

The authors demonstrated that turnover of Dam1-complex subunits was not taking place and that the ringstructure is sliding intact along the depolymerizing microtubule.

Finally, Barnes and colleagues showed that the Dam1 complex can function as a coupling device to move objects along themicrotubules. These experiments relied on the strong bonds formed between streptavidin and biotin molecules. Streptavidincoatedmicrobeads were recruited to the microtubules using a biotin-labelled Daml complex. As themicrotubules depolymerized, the Daml complex slid along the spindle away from the shrinking ends, bearing its cargo of beads. It will be interesting to find out whetherchromosomes are transported in the same way. DeepaNath the same quantum state), and the atomic-pair potential diverges steeply towards infinity as $r$ approaches zero. And at the mesoscopic scale - that between the microscopic and the macroscopic - the rigid core of colloidal particles ensures that they just 'bounce off' each other at low separations, and also do not overlap. Neither the atomic nor the mesoscopic potentials even possess a Fourier transform; for a long time, finite interactions of the type considered by Sütö ${ }^{\prime 1}$ were simply considered 'unphysical.

But effective potentials between the centres of mass of entities such as block copolymers ${ }^{2}$, polymer chains or dendrimers can indeed remain finite at zero separation (Fig. 1). Theory $^{3}$ and simulation ${ }^{4}$ have shown that the effective potential between two chains has a gaussian form, and calculations have shown that its ground-state configuration is facecentred cubic ( $\mathrm{fcc}$ ) at low densities and bcc at high densities ${ }^{5,6}$. Fourier transformation does not change a gaussian form, so $\hat{\varphi}(k)$ is positive in this case. There is, however, no value of $K_{0}$ above which $\hat{\varphi}(k)$ is zero, so Sütő's result is not valid here. Nevertheless, his work joins a rapidly growing body of research ${ }^{7}$, spurred on by developments in soft-matter physics, on the properties of bounded interaction potentials in one-component systems, in which the properties of $\hat{\varphi}(k)$ are used to obtain information on the ground-state configuration or the topology of the phase diagram.

A potential $\hat{\varphi}(k)$ that vanishes above a threshold seems difficult to realize physically. Süto shows that the requirement for this to occur leads to an interaction potential that shows oscillatory behaviour combined with a power-law decay at large values of $r$. This is strikingly similar to the RKKY (RudermanKittel-Kasuya-Yosida), or Friedel, potential $^{8-10}$ that arises as an effective interaction potential between ions when the effect of free electrons is incorporated as a statistical average. For metals of valency $Z$, the ion density $\rho$ is related to Süto's minimum density by $\rho=\rho^{*} \pi \sqrt{ } 2 /(3 Z)$, so that $\rho$ exceeds $\rho^{*}$ by $48 \%$ for $Z=1$ and trails it by $26 \%$ for $Z=2$. So unfortunately there is no happy agreement between the real and the Sütó densities that would hint at why certain metals crystallize into the boc structure. The power laws governing the behaviour of the RKKY and Süt $\delta$ potentials at large values of $r$ are slightly different, and effective ion potentials also include a steeply repulsive region at small values of $r$ (ref. 10) that is absent in Sütö's interaction. So the long- and short-range parts of the potential must be considered independently. Süto provides us with a suitable reference frame to deal with the former

In the late 1970 s, a celebrated and controversial article ${ }^{\text {I }}$ was published entitled 'Should all crystals be bcc?'. It contained a theory of freezing that involved density waves - modulated density profiles - at the set of reciprocal lattice vectors $K$ as parameters in a mathematical expression for the free energy. On the basis that the bcc crystal is the only spatial configuration that combines the shortest reciprocallattice vectors lying on the surface of a sphere, it was concluded that the stablest crystals should all be bcc. This argument, although brilliant, was flawed in making certain simplifying assumptions. Süt $\delta$ 's work comes from a very different direction to again put the bcc lattice in a distinctive position among all Bravais lattices. In this way, it recasts the question of why crystals form as they do in a fresh and thought-provoking way.

Christos N. Likos is at the Institut für Theoretische Physik II, Heinrich-HeineUniversităt Düsseldor f, Universitătsstraße 1, D-40225 Düsseldorf, Germany. e-mail:likos@thphy.uni-duesseldorf.de

1 SutäA.Phys Rev. Lett. 95, 265501(2005)

2. Plerleoni, C, Addison, C, Hansen,J-P.\& Krakoviack, V. Phys. Rex Lett. (in the press); preprint ava ilable at wwwarxineorg/abs/cond-mat/0601417 (2006)

3 Krüger, B, Schäfer, L.\& Baumgärthec, A.J. Phys. (Paris) 50, 391(1989).

4. Louls, A.A, Bolhuis, P. G, Hansen, 1.-P. \& Melijer, E. L. Phys Pev. Lett 85, 2522-2525(2000)

5. Stillinger, F. H. J.Chem. Phys 65,3968-3974 (1976)

6. Stillinger, F. H. Phys. Rex B20, 299-302(1979).

7. Mladek, B. M, Gottwald, D, Kahl, G, Neumann, M.\& Likos, C.N. Phys Rev. Lett. 96, 045701(2006).

8. Ashcroft, N.W.\& Mermin, N.D. SolldStatePhysics (Holt Saunders, Philadelphia, 1976).

9. Ziman, 1. M. Principles of the Theory of Salids (Cambridge Univ. Press, 1972)

10. Hafnec, L. Fram Hamitonians to Phase Diagrams (Springer, Berlin, 1987).

11. Alexander, S.\& McTague, L. Phys. Rex Lett. 41, 702-705 (1978). 